\title{
Comércio internacional " $x$ " intranacional no Brasil: medindo o efeito-fronteira
}

Orlando M. da Silva Professor do Departamento de Economia da UFV

Fernanda M. de Almeida Graduanda da UFV

Bethania M. de Oliveira Economista da Pemagram

\section{Palauras-chave}

comércio internacional, comércio intranacional, efeito-fronteira, modelo de gravidade.

Classificação JEL F10, F14, F15.

Key words

international trade, intranational trade, border effect, gravity model.

JEL Classification F10, F14, F15.

\section{Resumo}

Neste artigo, analisou-se, para o mercado brasileiro, o efeito-fronteira, que indica o viés do comércio doméstico em comparação com o comércio internacional. Esse efeito foi estimado empiricamente, utilizando-se dados de corte seccional, em um modelo de gravidade com os 26 estados brasileiros mais o Distrito Federal e 46 países. Apesar de o Brasil estar reduzindo as barreiras ao comércio internacional, o efeito-fronteira calculado mostrou-se ainda muito alto para os padrões conhecidos. O valor encontrado para o viés entre os estados brasileiros foi igual a 33, evidenciando-se que eles comercializam 33 vezes mais entre si do que com os países estrangeiros. Em um país em desenvolvimento como o Brasil, as conseqüências desse viés, em termos de bem-estar, provavelmente sejam muito grandes. A magnitude do efeitofronteira pode ser explicada pelo baixo grau de substituição entre os produtos domésticos e estrangeiros e, também, pelas grandes barreiras ao comércio internacional.

\begin{abstract}
This paper analyses the border effect on the Brazilian goods market, which indicates how biased intranational trade is compared to international trade. We quantify the border effect empirically using a cross-sectional gravity equation for twenty six Brazilian states plus the Federal District and forty-six countries, and find that although Brazil has been lowering its trade barriers, the border effect is still very high by any standards. The bias in a typical Brazilian state relative to other countries was found to be around 33, suggesting that trade among Brazilian states is thirty-three times as high as trade with a foreign country. The welfare consequences of a bias of this magnitude are likely to be very large for a developing country such as Brazil. It can be explained both by the low degree of substitutability among goods produced in Brazilian states and foreign countries, as well as by the large barriers to international trade.
\end{abstract}




\section{1_Introdução}

A interdependência econômica entre as nações tem aumentado substancialmente nas últimas cinco décadas, com o comércio internacional de bens e serviços expandido a taxas superiores às da produção. Também o fluxo financeiro internacional tem-se elevado muito e contribuído significativamente para a melhoria do padrão de vida da população em vários países. Essa maior integração é devida a fatores tecnológicos, sociais e culturais e ainda à mudança em muitas políticas governamentais e em governos que restringiam as transações internacionais.

Quando as barreiras entre os países eram altas, os governos e os cidadãos podiam distinguir imediatamente as políticas domésticas das internacionais; contudo, na medida em que elas foram sendo reduzidas e/ou eliminadas, tornou-se mais difícil distingui-las. A transmissão dos efeitos internacionais sobre a economia doméstica e a diminuição da autonomia e mesmo da soberania nacional, causadas pela maior integração dos países, têm requerido virtualmente uma análise econômica e política de todas as políticas não-comerciais. Foi com esse espírito que, a partir de meados da década de 1990, começou a surgir uma série de estudos sobre o relacionamento entre os comércios internacional e intranacional
(McCallum, 1995; Helliwell, 1996; Feenstra et al., 1998; Debaere, 2002). O estudo inicial se deveu a McCallum (1995), que utilizou dados de 1988 sobre o comércio entre as províncias do Canadá e dos Estados Unidos, mostrando que o comércio entre duas províncias canadenses era em torno de 20 vezes maior que o comércio dessas províncias com os Estados Unidos. Helliwell (1996), expandindo a amostra para o período de 1988-1994, confirmou os resultados de McCallum, indicando um grau alarmante de viés para os produtos domésticos em países com desenvolvimento igual ao dos Estados Unidos e do Canadá. Os modelos utilizados naqueles estudos se basearam na análise de fluxos bilaterais de comércio, por meio de "equações de gravidade" que visam explicar o comércio com variáveis como a proximidade geográfica, o tamanho econômico, ou os níveis de renda dos países. No Brasil, o estudo inicial de utilização do modelo para estimar impactos de variáveis sobre o comércio regional é o de Hidalgo e Vergolino (1998), que avaliaram as características do comércio do Nordeste com as outras macrorregiões brasileiras e com outros países, utilizando uma matriz de comércio de 1991. Os resultados obtidos indicaram que as exportações, para os demais Estados, mostraram-se 11 vezes maiores que as internacionais. 
Esse viés para as compras domésticas é uma medida do grau em que os mercados são segmentados pelas fronteiras políticas. Entre as várias razões para a sua existência, podem-se citar as barreiras comerciais (tarifárias e não-tarifárias), naturais (custo e tempo de transporte), culturais e institucionais, a exposição ao risco cambial, as preferências por produtos domésticos, existência de canais naturais de comércio entre os oligopólios, etc.

Aplicações do modelo gravitacional no Brasil como as de Piani e Kume (2000), Sá Porto (2002), Azevedo (2004) e Azevedo et al. (2006), avaliaram os efeitos do fluxo bilateral de comércio brasileiro, destacando o impacto de blocos ou regiões comerciais selecionados. Contudo, não enfatizaram o viés para compras internas, supostamente pela dificuldade da obtenção de dados sobre o comércio regional no Brasil. Atualmente, esses dados estão disponíveis por meio de estudos realizados pelo Instituto de Pesquisa Econômica Aplicada (Vasconcelos, 2001; Vasconcelos e Oliveira, 2006) e permitem a utilização dos modelos gravitacionais para cálculo do efeito-fronteira no Brasil. Assim, os objetivos deste estudo foram calcular e analisar o efeito-fronteira no comércio exterior brasileiro. Admite-se que o cálculo do efeito-fronteira para a economia brasileira seja importante ao estimular a análise política e econômica da sua ocorrência. Este estudo inova ao utilizar a mais recente fonte de dados sobre as exportações dos 26 Estados da Federação mais o Distrito Federal, além de utilizar, no modelo, variáveis como adjacência e similaridade de renda para captar diferentes efeitos do comércio intranacional.

\section{2_Metodologia}

$\mathrm{Na}$ sua forma mais simples, a equação de gravidade indica que o comércio bilateral entre dois países é diretamente proporcional ao produto de seus Produtos Internos Brutos (PIBs) e inversamente proporcional à distância entre eles. Assim, países grandes tendem a comercializar mais entre si, da mesma forma que aqueles mais similares em seu tamanho relativo. Entretanto, a distância entre eles funciona como uma "resistência" ao comércio, captando efeitos de variáveis como custo de transporte, de informação, etc. Tinbergen (1962) e Linnemann (1966) foram os pioneiros no uso das equações de gravidade para estimar fluxos de comércio. No início, as equações de gravidade eram estimadas assumindo que os países eram especializados em diferentes variedades de produtos, com demandas iguais e homotéticas, e que o comércio era livre, 
sem tarifas e custos de transporte. Assim, um produto produzido em qualquer país seria enviado aos demais na proporção dos PIBs dessas nações.

Helpman (1987) derivou uma equação de gravidade que considerava os produtos diferenciados, com retornos crescentes à escala (modelo de competição monopolística), além de chamar a atenção para a relação direta entre o PIB dos países e o volume de comércio.

Debaere (2002) testou a relação proposta por Helpman usando dados de séries temporais do período de 1970-1989, para uma amostra de países pertencentes e não-pertencentes à OCDE. Nos países da OCDE, ele comprovou a relação positiva entre o tamanho dos países e o comércio, mas, no grupo de países não-pertencentes à OCDE, os resultados foram bem diferentes. A explicação foi de que a equação de gravidade era baseada na suposição de que os países são especializados em produtos diferentes, o que é uma descrição razoável do comércio entre os países industrializados, mas não entre aqueles em desenvolvimento, que exportam basicamente produtos agrícolas ou "commodities". Nesses casos, não há razão para a equação de gravidade funcionar, e isso é o que foi encontrado nos países não pertencentes à OCDE.

Estudos subseqüentes utilizaram especificações diferentes, introduzindo no- vas variáveis e novos métodos de estimação. Uma boa revisão sobre os modelos gravitacionais e suas aplicações pode ser encontrada no trabalho de Paz (2003) e no Feenstra (2004).

A aplicação do modelo de gravidade, proposta por McCallum (1995) e que tem gerado grande quantidade de pesquisa, compara o comércio intranacional com o internacional. Ele estimou um modelo em que a variável dependente eram as exportações de cada província do Canadá para as outras províncias ou para os Estados Unidos. As exportações dependiam dos PIBs das províncias e das distâncias entre elas, de tal forma que a regressão estimada foi:

$$
\begin{aligned}
\ln X_{i j} & =\alpha+\beta_{1} \ln Y_{i}+\beta_{2} \ln Y_{j}+ \\
& +\beta_{3} \ln d_{i j}+\gamma \delta_{i j}+\varepsilon_{i j}
\end{aligned}
$$

sendo $\gamma$ uma variável "dummy", que assume o valor unitário para o comércio entre as províncias e zero, caso contrário. Os resultados encontrados por McCallum (1995), utilizando dados de 1988, e por Anderson e van Wincoop (2003), utilizando dados de 1993, indicaram coeficientes dos PIBs das províncias próximos da unidade e forte relacionamento negativo entre distância e comércio. $\mathrm{O}$ que não era esperado foi o valor encontrado para o coeficiente de comércio entre as províncias $(3,09 \mathrm{em} 1988$ e 2,75 em 
1993), apontando que o comércio entre elas era em torno de 22 vezes maior que o comércio internacional em 1988 e 16 vezes maior em 1993. Apesar de esses números captarem todo e qualquer fator impeditivo do comércio entre Canadá e Estados Unidos (efeito-fronteira), eles pareceram extremamente altos.

De acordo com Anderson e van Wincoop (2003), há dois problemas básicos na estimação da equação proposta por McCallum. O primeiro se refere a uma assimetria do efeito-fronteira entre países com tamanhos diferentes, com países menores apresentando efeitos maiores. $\mathrm{O}$ segundo e mais importante é que o modelo sofre do problema de omissão de variáveis denominadas "termos de resistência multilateral" (ex. custos de transporte e tarifas, que afetam os preços relativos), que levam a uma superestimação do efeito-fronteira. Segundo Feenstra (2004), diversas abordagens têm sido utilizadas para minimizar esse viés, como a de Bergstrand (1985) e Baier e Bergstrand (2001), que fizeram uso de índices de preços para captar os efeitos-preço; a de Anderson e van Wincoop (2003), que utilizaram "efeitos-preço" estimados; ou aquela de Redding e Venables (2000), que usaram "efeitos fixos" na derivação da equação de gravidade.

Para este estudo, variáveis de resistência multilateral não serão consideradas explicitamente no modelo. A inexistência de dados sobre os custos de transporte entre os diversos Estados brasileiros e de restrições comerciais (tarifas) entre eles leva à suposição de igualdade de preços internos para os produtos comercializados. Apesar de minimizado por essa pressuposição, admite-se ainda um viés positivo para o efeito-fronteira, decorrente não só da grande assimetria econômica existente entre os Estados brasileiros, mas também entre o Brasil e alguns de seus parceiros comerciais.

O estudo de Paz (2003), que utilizou um modelo Tobit com dados de corte seccional para 26 Estados brasileiros empilhados por ano, encontrou resultados bastante divergentes para o efeito-fronteira. Considerando as observações zero da amostra, os valores estimados para o efeito-fronteira foram exageradamente elevados. Quando as observações zero foram descartadas, o menor valor encontrado foi igual a 15 , indicando que o comércio interestadual é 15 vezes maior do que o internacional.

$O$ modelo a ser utilizado aqui inclui, além das variáveis tradicionais anteriormente citadas, aquelas que procuram captar os efeitos da adjacência e da similaridade da renda dos Estados nos padrões de comércio entre eles.

Assim, o modelo proposto tem a seguinte especificação na forma log-linear: 
$\ln X_{i j}=\beta_{0}+\beta_{1} \ln P I B_{i}+\beta_{2} \ln P I B_{j}+\beta_{3} \ln D_{i j}+\beta_{4} F C+\beta_{5} A d j+\beta_{6} \ln \left(P P C_{i}-P P C_{j}\right)^{2}+\mu_{i j}$

em que: $X_{i j}=$ as exportações do Estado $i$ para o Estado ou país $j$; $P I B_{k}=o$ PIB nominal dos Estados ou países $i$ e $j$; $D_{i j}=$ a distância em $\mathrm{km}$ do Estado $i$ ao Estado ou país $j$; $F C=$ uma variável "dummy" que assume o valor 1 se as exportações do Estado $i$ vão para o Estado $j$ do Brasil e zero se elas têm como destino outro país; $A d j=$ uma variável "dummy" que assume o valor 1 se as exportações do Estado $i$ vão para um Estado ou país adjacente e zero, caso contrário; $P P C_{i}-P P C_{j}=$ a diferença entre o PIB per capita entre os Estados e países;

$\beta_{0}$ a $\beta_{6}=$ parâmetros com sinais positivos, à exceção de $\beta_{3}$ e $\beta_{6}$; $\mu=$ o termo de erro.

Os dados utilizados são do ano de 1999. Aqueles sobre as exportações dos 26 Estados brasileiros e do Distrito Federal, uns para os outros e para os principais parceiros (46 países) comerciais, foram obti- dos de Vasconcelos e Oliveira (2006) e do site Aliceweb, do Ministério do Desenvolvimento, Indústria e Comércio Exterior (MDIC). A escolha dos 46 países se deu em função da existência de fluxos de comércio com os Estados naquele ano. Ao fazer essa opção, reduziu-se o número de observações zero da amostra, que passaram a existir somente para o comércio entre alguns dos Estados. Dados sobre o PIB e a população (nacional e por unidade da Federação) foram obtidos do Instituto Brasileiro de Geografia e Estatística (IBGE), enquanto aqueles dos países selecionados o foram do Penn World Table. Todos os valores monetários eram nominais e foram expressos em real. Os PIBs dos demais países foram transformados em real, utilizando-se a taxa de câmbio média (real/US\$), para o ano de 1999. As distâncias, medidas em quilômetros, foram obtidas do site Areaseg.com e representam as distâncias físicas entre as capitais de cada Estado. Nos demais países, considerou-se a distância dos Estados até Brasília e de lá até a capital de cada um dos países, sendo esses dados retirados do Centre d'Etudes Prospectives et d'Informations Internationales (CEPII). 


\section{Resultados}

$\mathrm{Na}$ Tabela 1 estão apresentados os resultados obtidos com a estimação do modelo proposto e de algumas de suas variações, utilizadas para verificar a estabilidade das estimativas. De maneira geral, os resultados foram muito bons, com as variáveis consideradas explicando mais de $60 \%$ das variações nas exportações estaduais. A coluna (1) indica a versão mais simples do modelo, as colunas (2) e (3) apresentam as estimativas considerando as variáveis "dummies" para fronteira exterior e adjacências, respectivamente, enquanto a coluna (4) considera, além dessas variáveis, um indicador de similaridade de renda entre os Estados e os países.

As colunas de (5) a (8) mostram os resultados das mesmas equações, substituindo-se as variáveis PIB pela população. Como em alguns casos (19 observações) não houve fluxo de comércio entre os Estados, decidiu-se fazer uma estimação do modelo com todas as observações, pelo método Tobit, cujos resultados são apresentados nas colunas (9) e (10).

A elasticidade encontrada para as exportações em relação ao PIB doméstico tem valor próximo da unidade e é coerente com as várias aplicações do modelo gravi- tacional. No caso do PIB dos Estados ou dos países importadores, o resultado médio encontrado para as elasticidades das exportações foi de 0,6 . Nas equações em que a variável PIB foi substituída pela variável população, os valores estimados para as elasticidades exportação foram um pouco maiores em relação à população doméstica (média $=1,3$ ) e um pouco menores em relação à população dos Estados ou dos países importadores (média $=0,35$ ).

Os coeficientes estimados para a variável distância foram sempre negativos e com elevado grau de significância estatística. Nas equações com a variável PIB, as elasticidades das exportações em relação às distâncias foram um pouco maiores, com um valor médio de $-0,85$.

Os valores dos coeficientes da variável "dummy" para o efeito-fronteira variaram entre 3,06 e 4,48, todos com elevada significância estatística. $O$ valores apresentados nas colunas de (2) a (4) indicam que as exportações interestaduais no Brasil são entre 33 e 38 vezes maiores do que aquelas para os demais países (exp $\left.\beta_{4}\right)$. Os coeficientes estimados quando a população é utilizada no lugar do PIB indicam que as exportações intranacionais são de 21 e 25 vezes maiores que as exportações internacionais. 
Tabela 1_ Estimativas do modelo de gravidade - Brasil, 1999

\begin{tabular}{|c|c|c|c|c|c|c|c|c|c|c|}
\hline \multirow{2}{*}{$\begin{array}{c}\text { Variável } \\
\text { Independente }\end{array}$} & \multicolumn{10}{|c|}{ Equações } \\
\hline & (1) & (2) & (3) & (4) & (5) & (6) & (7) & (8) & (9) & (10) \\
\hline Constante & $\begin{array}{l}-4,777 * \\
(1,866)\end{array}$ & $\begin{array}{c}-13,407^{*} \\
(1,290)\end{array}$ & $\begin{array}{c}-14,946^{*} \\
(1,310)\end{array}$ & $\begin{array}{c}-16,618^{*} \\
(1,297)\end{array}$ & $\begin{array}{c}3,663^{*} \\
(1,324)\end{array}$ & $\begin{array}{l}-2,051^{* *} \\
(1,087)\end{array}$ & $\begin{array}{c}-3,953^{*} \\
(1,113)\end{array}$ & $\begin{array}{l}-4,688^{*} \\
(1,099)\end{array}$ & $\begin{array}{c}-20,767^{*} \\
(1,184)\end{array}$ & $\begin{array}{l}-6,015^{*} \\
(0,908)\end{array}$ \\
\hline $\log \left(\mathrm{PIB}_{\mathrm{i}}\right)$ & $\begin{array}{c}0,974 * \\
(0,106)\end{array}$ & $\begin{array}{c}1,084^{*} \\
(0,061)\end{array}$ & $\begin{array}{c}1,086^{*} \\
(0,061)\end{array}$ & $\begin{array}{c}1,196^{*} \\
(0,061)\end{array}$ & & & & & $\begin{array}{c}1,222^{*} \\
(0,046)\end{array}$ & \\
\hline $\log \left(P I B_{j}\right)$ & $\begin{array}{c}0,389 * \\
(0,028)\end{array}$ & $\begin{array}{c}0,602 * \\
(0,029)\end{array}$ & $\begin{array}{c}0,578^{*} \\
(0,029)\end{array}$ & $\begin{array}{c}0,855^{*} \\
(0,047)\end{array}$ & & & & & $\begin{array}{c}1,065^{*} \\
(0,048)\end{array}$ & \\
\hline $\log \left(D_{i j}\right)$ & $\begin{array}{l}-1,094^{*} \\
(0,079)\end{array}$ & $\begin{array}{l}-0,950^{*} \\
(0,075)\end{array}$ & $\begin{array}{l}-0,729 * \\
(0,082)\end{array}$ & $\begin{array}{l}-0,651 * \\
(0,081)\end{array}$ & $\begin{array}{l}-0,818^{*} \\
(0,076)\end{array}$ & $\begin{array}{l}-0,623^{*} \\
(0,077)\end{array}$ & $\begin{array}{l}-0,387^{*} \\
(0,082)\end{array}$ & $\begin{array}{l}-0,524^{*} \\
(0,085)\end{array}$ & $\begin{array}{l}-0,667^{*} \\
(0,088)\end{array}$ & $\begin{array}{l}-0,896^{*} \\
(0,079)\end{array}$ \\
\hline $\log \left(P_{o p}{ }_{i}\right)$ & & & & & $\begin{array}{c}1,217^{*} \\
(0,141)\end{array}$ & $\begin{array}{c}1,358^{*} \\
(0,094)\end{array}$ & $\begin{array}{c}1,355^{*} \\
(0,094)\end{array}$ & $\begin{array}{c}1,294^{*} \\
(0,092)\end{array}$ & & $\begin{array}{c}1,373^{*} \\
(0,053)\end{array}$ \\
\hline $\log \left(P o p_{j}\right)$ & & & & & $\begin{array}{c}0,264^{*} \\
(0,027)\end{array}$ & $\begin{array}{c}0,419 * \\
(0,029)\end{array}$ & $\begin{array}{c}0,401 * \\
(0,029)\end{array}$ & $\begin{array}{c}0,331 * \\
(0,032)\end{array}$ & & $\begin{array}{c}0,688^{*} \\
(0,040)\end{array}$ \\
\hline $\log (\operatorname{SimPIB})$ & & & & $\begin{array}{l}-0,161 * \\
(0,022)\end{array}$ & & & & $\begin{array}{c}0,081^{*} \\
(0,015)\end{array}$ & $\begin{array}{l}-0,170^{*} \\
(0,025)\end{array}$ & $\begin{array}{c}0,088^{*} \\
(0,017)\end{array}$ \\
\hline Dummy FC & & $\begin{array}{c}3,486^{*} \\
(0,185)\end{array}$ & $\begin{array}{c}3,512^{*} \\
(0,184)\end{array}$ & $\begin{array}{c}3,633^{*} \\
(0,181)\end{array}$ & & $\begin{array}{c}3,059 * \\
(0,209)\end{array}$ & $\begin{array}{c}3,093^{*} \\
(0,208)\end{array}$ & $\begin{array}{c}3,210^{*} \\
(0,204)\end{array}$ & $\begin{array}{c}4,388^{*} \\
(0,145)\end{array}$ & $\begin{array}{c}3,699 * \\
(0,155)\end{array}$ \\
\hline Dummy Adj & & & $\begin{array}{c}1,166^{*} \\
(0,178)\end{array}$ & $\begin{array}{c}1,181^{*} \\
(0,175)\end{array}$ & & & $\begin{array}{c}1,325^{*} \\
(0,185)\end{array}$ & $\begin{array}{c}1,264^{*} \\
(0,185)\end{array}$ & $\begin{array}{c}1,165^{*} \\
(0,289)\end{array}$ & $\begin{array}{c}-0,758^{*} \\
(0,183)\end{array}$ \\
\hline $\mathrm{R}^{2}$ Ajustado & 0,639 & 0,695 & 0,704 & 0,715 & 0,612 & 0,653 & 0,665 & 0,672 & - & 一 \\
\hline $\mathrm{F}$ & 590,49 & 608,34 & 529,85 & 480,01 & 526,81 & 502,98 & 443,44 & 391,17 & 一 & - \\
\hline Num. Obs. & 1334 & 1334 & 1334 & 1334 & 1334 & 1334 & 1334 & 1334 & 1353 & 1353 \\
\hline Método Est. & MQO & $\mathrm{MQO}$ & MQO & MQO & MQO & MQO & MQO & MQO & Tobit & Tobit \\
\hline
\end{tabular}

Obs:: Os valores entre parêntesis são os erros-padrão das estimativas. 
Esses resultados estão em sintonia com aqueles encontrados por Paz (2003), que, estimando um modelo similar com dados de 1991 e um modelo com dados em painel para os anos de 1991, 1997, 1998 e 1999, encontrou valores para o efeitofronteira que variaram entre 15 e 1.293 . Chama-se a atenção para o fato de que, ao utilizar um número maior de países e dados dos anos de 1991, 1997, 1998 e 1999, aquele autor usou mais de 50 por cento das 21.000 observações com valores zero, o que contribuiu para aumentar o efeito-fronteira estimado. Para o caso deste estudo, pode-se notar que, apesar de terem sido utilizadas somente 19 observações zero, as estimativas obtidas pelo método Tobit são maiores que as demais.

Há de se considerar também o efeito da omissão dos termos de resistência multilaterais, principalmente os custos de transporte e a assimetria existente entre o comércio dos Estados brasileiros com os principais parceiros comerciais. $\mathrm{O}$ menor tamanho da economia brasileira relativo aos principais parceiros certamente contribui para o elevado efeito-fronteira estimado.

A estimação do efeito adjacência no comércio intranacional está indicada nas colunas (3), (4), (7) e (8), por coeficientes que variam de 1,17 a 1,32. Mantendo constantes as demais variáveis, esses valores in- dicam que o comércio de um Estado com outro ou país adjacente é de 3,2 a 3,7 vezes maior que as exportações para aqueles Estados ou países que não têm fronteira em comum. Esse também é um resultado surpreendente, dada a inexistência de barreiras tarifárias, mas que encontra alguma explicação na extensão territorial do Brasil e nas dificuldades de transporte entre os Estados. É importante ressaltar, contudo, que as equações que contêm a variável "dummy" para adjacência foram as que apresentaram melhores coeficientes de ajustamento e maiores valores para o efeito-fronteira, principalmente quando o PIB foi utilizado.

A hipótese de Linder de que os Estados ou países com renda per capita similar tendem a comercializar mais entre si do que aqueles Estados ou países com renda per capita diferentes foi testada com a inclusão do logaritmo da diferença das rendas per capita nas equações (4) e (8). O valor encontrado para o coeficiente de similaridade da renda na equação (4) foi negativo $(-0,16)$ e estatisticamente significante, indicando que os Estados com renda similar comercializam mais uns com os outros, como esperado. Contudo, na equação (8), quando a variável população dos Estados substitui o PIB, o coeficiente estimado foi positivo, apesar de muito pequeno $(0,08)$, 
contrariando a previsão de Linder. As grandes diferenças econômicas e estruturais dos Estados brasileiros permitem um resultado como esse, principalmente pelo comércio do tipo interindústria entre eles e com o mercado internacional.

As colunas (9) e (10) apresentam os resultados do modelo estimado com todas as variáveis, utilizando o método Tobit, em função da natureza censurada da variável dependente em 19 observações. Todos os coeficientes indicaram os sinais esperados e são estatisticamente significantes, com exceção da variável adjacência da equação com população, na coluna (10). Os valores dos coeficientes obtidos são condizentes com aqueles estimados utilizando MQO.

\section{Conclusões}

Utilizando variantes do modelo gravitacional, este estudo procurou mensurar o efeito-fronteira no mercado brasileiro. Para tanto, utilizou-se de uma matriz de comércio que incluiu os 27 Estados brasileiros e 40 países, perfazendo um total de 1353 observações.

O efeito-fronteira estimado reflete todos os fatores que contribuem para que o comércio interno do Brasil seja maior do que aquele com outros países, e não somente o efeito de barreiras tarifárias e não-tarifárias.
Como previsto, o efeito doméstico se mostrou consistente com outros estudos dessa natureza, quando a elasticidade-renda (PIB) doméstica das exportações foi maior do que aquela dos demais Estados e países. Também o valor médio da elasticidade distância das exportações permite concluir que aumento de $1 \%$ nas distâncias reduziria as exportações em $0,72 \%$.

Os valores obtidos para o efeitofronteira indicam que o comércio intranacional no Brasil é, em média, 33 vezes maior que aquele com os países. Não resta dúvida de que a assimetria econômica entre os Estados brasileiros e outros países de tamanho econômico muito diferente contribui para esse efeito, que é muito alto para qualquer padrão.

O comércio intranacional no Brasil apresenta forte concentração, com a maioria das exportações e importações realizadas por um pequeno número de Estados. Essa desigualdade de comércio se dá em função da elevada concentração da atividade econômica (principalmente industrial) em poucos Estados. O Estado de São Paulo, sozinho, foi o responsável por 35\% das exportações interestaduais, enquanto o Distrito Federal e os 21 Estados restantes das regiões Norte, Nordeste, Centro-Oeste exportaram conjuntamente $42 \%$ do total. Em relação ao PIB total, essas mesmas proporções são de $50 \%$ e 35\%, respectivamente. 
A concentração e assimetria econômica no País tornam os comércios intranacional e internacional brasileiros do tipo interindústria, pela baixa substituição entre os produtos produzidos nos diferentes Estados, tornando bastante significativas as perdas em termos de bem-estar, que são inversamente proporcionais ao grau de substitutabilidade dos produtos. Apesar da inexistência de barreiras tarifárias entre os Estados, o grau de substituição é baixo e magnificado pelos problemas estruturais ainda existentes no transporte e na comunicação entre eles. São esses problemas que fazem que os Estados adjacentes comercializem até 3,7 vezes mais, uns com os outros, do que aqueles que não o são. A comprovação aparente da hipótese de Linder de que Estados com rendas per capita similares tendem a comercializar mais entre si pode também ser explicada pela concentração similar do PIB e das exportações na economia brasileira.

Um estudo como este pode ser ampliado, com a adição de variáveis que reflitam a dotação regional de recursos e a assimetria econômica, além de variáveis que captem, para um país continental como o Brasil, uma medida mais apropriada de distância interna. Sugere-se também o acompanhamento temporal do comércio intranacional e a utilização de métodos mais flexíveis de estimação. 


\section{Referências bibliográficas}

\author{
ANDERSON, James A.; Eric van \\ WINCOOP. Gravity with \\ gravitas: a solution to a border \\ puzzle. American Economic Review, \\ v. 93, n. 1, p. 170-192, 2003.
}

\section{AREASEG.COM. Distâncias entre} as capitais brasileiras. Disponível em:<http://www.areaseg.com /distancias.html>. Acesso em: 29 ago. 2006.

AZEVEDO, André F. Z. O efeito do Mercosul sobre o comércio: uma análise com o modelo gravitacional. Pesquisa e Planejamento Econômico, v. 34, n. 2, p. 307-339, 2004.

AZEVEDO, André F. Z.; PORTUGAL, Marcelo S.; BARCELLOS NETO, Paulo C. F. Impactos comerciais da Área de Livre Comércio da Américas: uma aplicação do modelo gravitacional. Revista de Economia Contemporânea, v. 10, n. 2 , p. $237-267,2006$.

BAIER, Scott; BERGSTRAND, Jeffrey $H$. The growth of world trade: tariffs, transport costs, and income similarity. Journal of International Economics, v. 53, n. 1 , p. 1-27, 2001.
BERGSTRAND, Jeffrey H. The generalized gravity equation, monopolistic competition, and the factor proportion theory in international trade. Review of Economics and Statistics, v. 71, n. 1 p. $43-153,1985$

\section{CENTRE D'ETUDES}

PROSPECTIVES ET

D'INFORMATIONS

INTERNATIONALES - CEPII.

Databases - Distance. Disponível

em: <http://www.cepii.fr/ anglaisgraph/bdd/distances.htm $>$. Acesso em: 4 nov. 2006

DEBAERE, Peter. Testing "new" trade theory with testing for gravity: re-interpreting the evidence. Austin: University of Texas, 2002. Manuscrito.

FEENSTRA, Robert C. Advanced international trade: theory and evidence. Princeton: Princeton University Press, 2004. 484 p.

FEENSTRA, Robert C; MARKUSEN, James R; ROSE, Andrew K. Understanting the home market effect and the gravity equation: the role of differentiating goods. 1998 (NBER Working paper, 6804).

HELLIWELL, John F. How much do national borders matter?

Whashington, D.C.: Brookings Institution Press, 1996. 156 p.
HELPMAN, Elhanan. Imperfect competition and international trade: evidence from fortheen industrial countries. Journal of the Japanese and Internacional Economics, v. 1, n. 1, p. 62-81, 1987.

HIDALGO, Álvaro B.; VERGOLINO, José R. O Nordeste e o comércio inter-regional e internacional: Um teste dos impactos por meio do modelo gravitacional. Economia Aplicada, v. 2, n. 4, p. 707-725, 1998

INSTITUTO BRASILEIRO DE GEOGRAFIA E ESTATÍSTICA - IBGE. Pib por Unidade da Federação. Disponível em: <www.ibge.gov.br>. Acesso em: 15 set. 2006

INSTITUTO DE PESQUISA ECONÔMICA E APLICADA IPEA. Ipeadata. Disponível em: <www.ipeadata.gov.br> Acesso em: 3 set. 2006.

LINNEMANN, H. An econometric study of international trade flows. Amsterdam: North Holland, 1966.

McCALLUM, John. National borders matter: Canadá-US. regional trade patterns. American Economic Review, v. 85, n. 3, p. $615-23,1995$.

\section{MINISTÉRIO DO}

DESENVOLVIMENTO, INDÚSTRIA E COMÉRCIO EXTERIOR. Estatísticas da balanca comercial dos estados. Disponível em: $<$ http://www.desenvolvimento. gov.br>. Acesso em: 8 set. 2006.

PAZ, Lourenço Senne. Brazilian international and inter-state trade flows: an exploratory analysis using the gravity model. 2003. 93p Dissertação (Mestrado) - Escola de Pós-Graduação em Economia FGV/RJ, Rio de Janeiro, 2003.

PENN WORLD TABLE.

Database. Disponível em: <http://pwt.econ.upenn.edu/>. Acesso em: 10 out. 2006

PIANI, Guida; KUME, Honório Fluxos bilaterais de comércio e blocos regionais: uma aplicação do modelo gravitacional. Rio de Janeiro: IPEA, jul. 2000. (Texto para Discussão, 749).

REDDING, Stefhen; VENABLES, Anthony J. Economic geography and international inequality. [s.1.]: Center for Economic Policy Research, 2000. (Discussion paper, 2568). 
SÁ PORTO, P. C. Mercosul and regional development in Brazil: a gravity model approach. Estudos Econômicos, v. 32, n. 1, p. 125-153, 2002.

TINBERGEN, Jan. Shaping the world economy: suggestions for an international economy policy. New York: Twentieth Century Fund, 1962.

VASCONCELOS, José R. Matriz de fluxo do comércio interestadual no Brasil - 1998. Rio de Janeiro: IPEA, mar. 2001. (Texto para Discussão, 783).

VASCONCELOS, José R.; OLIVEIRA, Márcio Augusto.

Análise da matriz de fluxo do comércio interestadual no Brasil - 1999. Rio de Janeiro: IPEA, jul. 2006.

(Texto para Discussão, 1159).

\footnotetext{
Os autores agradecem a dois - pareceristas anônimos, pelos - valiosos comentários e sugestões, : eximindo-os de quaisquer erros - porventura remanescentes, - e à FAPEMIG, pelo apoio - financeiro. Versão preliminar deste . - artigo foi apresentada no XLV - Congresso da SOBER, em - Londrina, PR, em julho de 2007. 